\title{
Cytogenetics of species of the families Pimelodidae and Rhamdiidae (Siluriformes)
}

\author{
Ana Cláudia Swarça, Lucia Giuliano Caetano and Ana Lúcia Dias
}

\begin{abstract}
Only 33 species among about 300 belonging to the families Pimelodidae and Rhamdiidae have been studied cytogenetically. The diploid number varies from $2 n=46$ to $2 n=63$ chromosomes, with the karyotypes often being of the meta/submetacentric type. As a result, there is generally a very elevated fundamental number.
\end{abstract}

\section{INTRODUCTION}

The family Pimelodidae is the most diversified of the neotropical Siluriformes, with about 300 species in 50-60 genera (Mees, 1974). Although several taxonomic revisions of this family have appeared, the systematics of these genera is still confused.

The subfamily Rhamdiinae was recently elevated to the category of family by Pinna (1993), based on a phylogenetic study of the order Siluriformes, which demonstrated the polyphyletic nature of the traditional family Pimelodidae. Some genera of the family Pimelodidae, including Pimelodella, Rhamdia, Imparfinis, Cetopsorhamdia and Rhamdella, were grouped into the new family Rhamdiidae based on morphological characters. Similarly, the subfamily Pseudopimelodinae is now considered as the family Pseudopimelodidae, and contains the genera Microglanis and Lophyosilurus (Pinna, 1993).

The current classification of the families Pimelodidae, Rhamdiidae and Pseudopimelodidae is therefore:

Superorder: Ostariophysi

Order: Siluriformes

Sub-Order: Eusiluroidei

Family: Pimelodidae

Sub-Order: Rhamdioidei

Family: Rhamdiidae

Sub-Order: Loricarioidei

Family: Pseudopimelodidae

This classification was adopted in the present work, which summarizes cytogenetic data for species of the families Pimelodidae and Rhamdiidae mainly found in unpublished thesis and congress abstracts. The diploid number, haploid number, the presence of $\mathrm{B}$ chromosomes and other relevant data were examined (Tables I and II). The classification of the hydrographic basin was performed as described by Vari (1992).

\section{DISCUSSION}

The chromosomal number among the 33 species of the families Pimelodidae and Rhamdiidae studied cytogenetically ranged from $2 n=46$ to $2 n=63$ (Tables I and II). For a more conclusive analysis, the FN of some species of the two families was recalculated (in parentheses) and to others species the FN was calculated for the first time in the present study (asterisk), considering M and SM with 2 arms and ST-A with $1 \mathrm{arm}$. In some species it was not possible to describe the FN, and they were therefore not karyotyped. A relatively high fundamental number was observed in the two families (from 78 to 116) (Tables I and II), with a frequently asymmetrical karyotype that included elements with one or two arms and a preponderance of metacentric chromosomes.

The extensive karyotypic variability between these two families suggests that chromosomal readjustments were involved in speciation within this group, as proposed by Oliveira et al. (1988). In the family Pimelodidae, the genera Bergiaria, Iheringichthys, Parapimelodus, Pimelodus, Hemisorubim, Pseudoplatystoma, Sorubim and Paulicea have a diploid number of $2 \mathrm{n}=56$ chromosomes (Toledo and Ferrari, 1976b; Costa and Reggi, 1986; Dias and Foresti, 1990, 1993; Garcia et al., 1990; Souza et al., 1992; Fenocchio and Bertollo, 1992; Fenocchio, 1993; Fenocchio et al., 1994; Vissotto, 1995; Abucarma and Martins-Santos, 1996; Martins-Santos et al., 1996; Silva et al., 1996; Swarça, 1998; Marques et al., 1998), exceptions being Pimelodus blochii with 2n $=58$ (Della-Rosa et al., 1980) and the genera Callophysus and Pinirampus with $2 \mathrm{n}=50$ chromosomes (Gil, 1993; Vasconcelos, 1994; Swarça, 1998). The diploid number of 56 chromosomes seems to be characteristic for the Pimelodidae family; however, the basic number varied from 86 to 106 (Table I). The genus Pimelodus, besides displaying a conservation in the diploid 
Table I- Cytogenetic data for the family Pimelodidae.

\begin{tabular}{|c|c|c|c|c|c|c|c|c|c|c|}
\hline Genera, Species & Locality & $\mathrm{HB}$ & $\mathrm{n}$ & $2 \mathrm{n}$ & Karyotype & FN & Bs & Size B & HS & References \\
\hline \multicolumn{11}{|l|}{ Bergiaria } \\
\hline B. westermanni & R. São Francisco, MG & $\mathrm{SF}$ & & 56 & $42 \mathrm{~m}, \mathrm{sm}+14 \mathrm{st}$ & 98 & $0-5$ & small & & Dias and Foresti (1993) \\
\hline \multicolumn{11}{|l|}{ Callophysus } \\
\hline C. macropterus & R. Negro; R. Solimões, AM & AM & & 50 & $22 \mathrm{~m}+18 \mathrm{sm}+10 \mathrm{a}$ & (90) & & & & Gil(1993) \\
\hline \multicolumn{11}{|l|}{ Iheringichthys } \\
\hline I. labrosus & R. Mogi-Guaçu, SP & UP & & 56 & $26 m+14 s m+12 s t+4 a$ & $96^{*}$ & $0-2$ & micro & & Dias and Foresti (1990) \\
\hline I. labrosus & R. Paraná, PR & UP & & 56 & $42 \mathrm{~m}, \mathrm{sm}+14 \mathrm{st}, \mathrm{a}$ & 98 & & & & Garcia et al. (1990) \\
\hline I. labrosus & R. Tibagi, PR & UP & & 56 & $18 m+16 s m+12 s t+10 a$ & $90^{*}$ & $0-1$ & micro & & Silva et al. (1996) \\
\hline I. labrosus & D. Jurumirim, SP & UP & & 56 & $22 m+18 s m+10 s t+6 a$ & 96 & $0-1$ & micro & & Vissotto (1995) \\
\hline \multicolumn{11}{|l|}{ Parapimelodus } \\
\hline P. valenciennes & R. Guaíba, RS & PA & & 56 & & & & & & Costa and Reggi (1986) \\
\hline \multicolumn{11}{|l|}{ Pimelodus } \\
\hline P. blochii & R. Solimões, AM & $\mathrm{AM}$ & & 58 & & & & & & Della-Rosa et al. (1980) \\
\hline P. clarias & Argentina & PA & & 56 & & & & & & Fenocchio et al. (1994) \\
\hline P. clarias & Uruguai & $\mathrm{PA}$ & 26 & & & & & & & Gonzales (1994) \\
\hline P. fur & & & & 56 & $30 m+14 s m+12 a$ & $100^{*}$ & & & & Toledo and Ferrari (1976b) \\
\hline P. maculatus & & & & 56 & $30 m+14 s m+12 a$ & $100 *$ & & & & Toledo and Ferrari (1976b) \\
\hline P. maculatus & R. São Francisco, MG; R. Mogi-Guaçu, SP & $\mathrm{SF} ; \mathrm{UP}$ & & 56 & $40 \mathrm{~m}, \mathrm{sm}+16 \mathrm{st}, \mathrm{a}$ & 96 & & & & Dias and Foresti (1993) \\
\hline P. maculatus & R. Guaíba, RS & PA & & 56 & & & & & & Costa and Reggi (1986) \\
\hline P. maculatus & R. Tibagi; R. Congonhas, PR & UP & & 56 & $20 m+20 s m+10 s t+6 a$ & (96) & & & & Swarça (1998) \\
\hline P. maculatus & R. Sapucaí; D. Furnas, MG & UP & & 56 & $40 \mathrm{~m} / \mathrm{sm}+16 \mathrm{st} / \mathrm{a}$ & 96 & & & & Marques et al. (1998) \\
\hline P. maculatus & R. Paranapanema; D. Jurumirim, SP & UP & & 56 & $20 m+20 s m+10 s t+6 a$ & 96 & & & & Vissotto (1995) \\
\hline P. ornatus & R. Paraná, PR & UP & & 56 & $18 m+22 s m+6 s t+10 a$ & $96^{*}$ & & & & Abucarma and Martins-Santos 1996) \\
\hline$P$ sp. & & & & 56 & $30 m+14 s m+12 a$ & $100^{*}$ & & & & Toledo and Ferrari (1976b) \\
\hline$P$. sp. & & & 26 & & & & & & & Scheel (1973) \\
\hline$P$. sp. & R. São Francisco, MG & $\mathrm{SF}$ & & 56 & $40 \mathrm{~m}, \mathrm{sm}+16 \mathrm{st}, \mathrm{a}$ & $96^{*}$ & & & & Dias and Foresti (1993) \\
\hline \multicolumn{11}{|l|}{ Pinirampus } \\
\hline P. pirinampu & R. Paraná, PR & UP & & 50 & $22 m+12 s m+4 s t+12 a$ & (84) & & & & Vasconcelos (1994) \\
\hline P. pirinampu & R. Tibagi, PR & UP & & 50 & $26 m+12 s m+2 s t+10 a$ & (88) & & & & Swarça et al. (1999) \\
\hline \multicolumn{11}{|l|}{ Hemisorubim } \\
\hline H. platyrhynchos & R. Paraná, PR & UP & & 56 & $22 m+18 s m+6 s t+10 a$ & (96) & & & & Martins-Santos et al. (1996) \\
\hline \multicolumn{11}{|l|}{ Pseudoplatystoma } \\
\hline P. coruscans & R. Paraná, PR & UP & & 56 & $18 m+16 s m+10 s t+12 a$ & (80) & & & & Martins-Santos et al. (1996) \\
\hline P. coruscans & Coxim, MS & PA & & 56 & $42 \mathrm{~m}, \mathrm{sm}+14 \mathrm{st}, \mathrm{a}$ & (98) & & & & Souza et al. (1992) \\
\hline P. coruscans & R. Mogi-Guaçu, SP & UP & & 56 & $18 m+18 s m+10 s t+10 a$ & $92 *$ & & & & Bigoni et al. (1992) \\
\hline P. coruscans & D. Três Marias, MG & $\mathrm{SF}$ & & 56 & $20 m+12 s m+12 s t+12 a$ & (88) & & & & Fenocchio (1993) \\
\hline P. fasciatum & R. Solimões, AM & $\mathrm{AM}$ & & 56 & $18 m+14 s m+10 s t+14 a$ & (88) & & & & Fenocchio and Bertollo (1992) \\
\hline P. tigrinum & R. Solimões, AM & $\mathrm{AM}$ & & 56 & $18 m+16 s m+8 s t+14 a$ & (90) & & & & Fenocchio and Bertollo (1992) \\
\hline \multicolumn{11}{|l|}{ Paulicea } \\
\hline Paulicea luetkeni & R. Paraná & UP & & 56 & $26 m+10 s m+6 s t+14 a$ & (92) & & & & Martins-Santos et al. (1996) \\
\hline \multicolumn{11}{|l|}{ Sorubim } \\
\hline S. lima & R. Solimões, PR & $\mathrm{AM}$ & & 56 & $18 m+12 s m+14 s t+12 a$ & 86 & & & & Fenocchio and Bertollo (1992) \\
\hline S. lima & R. Paraná, PR & UP & & 56 & $20 m+14 s m+10 s t+12 a$ & (90) & & & & Martins-Santos et al. (1996) \\
\hline
\end{tabular}

$\mathrm{HB}=$ Hydrographic basin $; \mathrm{n}=$ haploid number; $2 \mathrm{n}=$ diploid number; $\mathrm{FN}=$ fundamental number $; \mathrm{B}=$ supernumerary chromosomes; HS = heterogametic sex; $\mathrm{m}=$ metacentrics; sm = submetacentrics; $\mathrm{st}=$ subtelocentrics; $\mathrm{a}=$ acrocentrics; $\mathrm{R}=$ river; $\mathrm{b}=$ brook; $\mathrm{L}=$ lagoon; $\mathrm{S}=$ stream; $\mathrm{D}=$ dam; $\mathrm{PA}=$ Paraguay; UP = Upper Paraná; $\mathrm{SF}=\mathrm{São}$ Francisco; AM = Amazon; MG, AM, SP, PR, RS, MS, BA = Brazilian states; ( ) FN recalculated; * FN calculated in the present study. 
Table II - Cytogenetic data for the family Rhamdiidae

\begin{tabular}{|c|c|c|c|c|c|c|c|c|c|c|}
\hline Genera, Species & Locality & $\mathrm{HB}$ & $\mathrm{n}$ & $2 \mathrm{n}$ & Karyotype & FN & Bs & Size B & HS & References \\
\hline \multicolumn{11}{|l|}{ Pimelodella } \\
\hline P. aff. gracilis & R. Tibagi, PR & UP & & 52 & $24 m+18 s m+4 s t+6 a$ & (94) & & & & Swarça (1998) \\
\hline P. kronei & Iporanga, $\mathrm{SP}$ & UP & & 58 & $54 \mathrm{~m}, \mathrm{sm}+4 \mathrm{st}$ & (112) & 1 & micro & & Almeida-Toledo et al. (1992) \\
\hline P. transitoria & Iporanga, SP & UP & & 58 & $54 \mathrm{~m}, \mathrm{sm}+4 \mathrm{st}$ & (112) & & & & Almeida-Toledo et al. (1992) \\
\hline$P$. sp. & R. Mogi-Guaçu, SP & UP & & 46 & $40 \mathrm{~m}, \mathrm{sm}+6 \mathrm{st}, \mathrm{a}$ & 86 & & & $\mathrm{XY}$ & Dias and Foresti (1993) \\
\hline$P$. sp. & R. Mogi-Guaçu, R. Pardo, SP & UP & & 46 & $28 m+10 s m+8 a$ & 84 & & & & Toledo and Ferrari (1976a) \\
\hline$P$. sp. & R. Araquá, SP; R. Capivara, SP & UP & & 46 & $32 \mathrm{~m}, \mathrm{sm}+14 \mathrm{st}, \mathrm{a}$ & $78^{*}$ & & & & Braga (1989) \\
\hline$P$. sp. & Argentina & PA & & 46 & & & & & & Fenocchio et. al (1994) \\
\hline$P$. sp. & R. Araquá, R. Capivara, SP & UP & & 46 & $20 m+20 s m+6 s t$ & 86 & & & & Vissotto (1995) \\
\hline$P$. sp. & R. Tibagi, PR & UP & & 46 & $34 \mathrm{~m}, \mathrm{sm}+12 \mathrm{st}, \mathrm{a}$ & $80^{*}$ & & & & Silva et al. (1996) \\
\hline$P$. sp. & R. Paraná, PR & UP & & 46 & $20 m+20 s m+6 s t$ & (86) & & & & Vasconcelos (1994) \\
\hline$P$. sp. & R. Paraná, PR & UP & & 52 & $22 m+22 s m+8 s t$ & (96) & & & & Vasconcelos (1994) \\
\hline$P$. sp. & R. Tibagi & UP & & 46 & $34 m+12 s m$ & (92) & & & & Swarca (1998) \\
\hline \multicolumn{11}{|l|}{ Rhamdella } \\
\hline$R$. sp. & Itaetê, BA & $\mathrm{SF}$ & & 56 & $26 \mathrm{~m}, \mathrm{sm}+30 \mathrm{st}, \mathrm{a}$ & $82 *$ & & & & Souza et al. (1994) \\
\hline \multicolumn{11}{|l|}{ Cetopsorhamdia } \\
\hline C. cf. iheringi & D. Três Marias, MG & $\mathrm{SF}$ & & 58 & $22 m+16 s m+10 s t+10 a$ & $96^{*}$ & & & & Fenocchio (1993) \\
\hline C. sp. & b. Canta Galo, SP & UP & & 58 & $22 \mathrm{~m}+18 \mathrm{sm}+10 \mathrm{st}+8 \mathrm{a}$ & $98^{*}$ & & & & Fenocchio (1993) \\
\hline C. sp. & R. Capivara, R. Pardo, SP & UP & & 58 & $28 m+24 s m+6 s t$ & 110 & & & & Vissotto (1995) \\
\hline \multicolumn{11}{|l|}{ Rhamdia } \\
\hline R. hilarii & R. Onça, SP & UP & & 62 & $36 m+18 s m+8 a$ & 116 & & & & Toledo and Ferrari (1976a) \\
\hline R. hilarii & D. Monjolinho, SP & UP & & 58 & & $>100$ & $0-5$ & small & & Fenocchio and Bertollo (1990) \\
\hline R. hilarii & D. Lobo, SP & UP & & 58 & & $>100$ & $0-3$ & small & & Fenocchio (1993) \\
\hline R. hilarii & D. $29, \mathrm{SP}$ & UP & & 58 & & $>100$ & $0-5$ & small & & Fenocchio (1993) \\
\hline R. hilarii & R. Mogi-Guaçu, SP & UP & & 58 & & $>100$ & & & & Fenocchio (1993) \\
\hline R. hilarii & R. São Francisco, MG & $\mathrm{SF}$ & & 58 & & $>100$ & $0-2$ & small & & Fenocchio (1993) \\
\hline R. hilarii & R. Aguapey, Corrientes, Argentina & PA & & 58 & & $>100$ & & & & Fenocchio (1993) \\
\hline R. hilarii & L. Nova; L. Jataí, SP & UP & & 58 & & $>100$ & $0-2$ & small & & Fenocchio (1993) \\
\hline R. hilarii & Center of Aquiculture-UNESP, Jaboticabal, SP & UP & & 58 & $58 \mathrm{~m}, \mathrm{sm}$ & $116^{*}$ & $0-2$ & medium & & Maistro (1996) \\
\hline$R . \mathrm{sp}$ & $\begin{array}{l}\text { b. Jacutinga, R. Araquá, R. Pardo, S. Quinta, } \\
\text { S. Hortelã, S. Jurumirim, SP }\end{array}$ & UP & & 58 & $30 m+18 s m+10 s t$ & 106 & $0-3$ & small & & Vissotto (1995) \\
\hline$R . \mathrm{sp}$. & R. Iguacu, Usina de Salto Segredo, PR & UP & & 58 & $36 m+14 s m+4 s t+4 a$ & (108) & $0-1$ & small & & Abucarma (1998) \\
\hline$R$. sp. & Sapucaí, D. Furnas, MG & UP & & $58-$ & & & $0-3$ & & & Andrade et al. (1998) \\
\hline R. branneri & R. Iguaçu, Usina de Salto Segredo, PR & UP & & 58 & $36 m+14 s m+4 s t+4 a$ & (108) & $0-2$ & medium & & Abucarma (1998) \\
\hline R. voulezi & R. Iguaçu, Usina de Salto Segredo, PR & UP & & 58 & $36 m+14 s m+4 s t+4 a$ & (108) & $0-1$ & medium & & Abucarma (1998) \\
\hline R. quelen & R. Iguaçu, PR & UP & & 58 & & & $0-1$ & small & & Fenocchio (1993) \\
\hline R. quelen & R. Paraná, Posadas, Argentina & PA & & 58 & & & & & & Fenocchio (1993) \\
\hline R. quelen & L. Nova and Jataí, SP & UP & & 58 & & & $0-4$ & small & & Fenocchio (1993) \\
\hline R. quelen & R. Guaíba; L. dos Quadros, RS & PA & & 58 & $52 \mathrm{~m}, \mathrm{sm}, \mathrm{st}+6 \mathrm{a}$ & 110 & $0-1$ & small & & Hochberg and Erdtmann (1988) \\
\hline R. sapo & Buenos Aires, Argentina & $\mathrm{PA}$ & & 58 & $44 \mathrm{~m}, \mathrm{sm}+14 \mathrm{st}, \mathrm{a}$ & 102 & $0-1$ & medium & & Valcarcel et al. (1993) \\
\hline R. sapo & Uruguai & $\mathrm{PA}$ & 28 & 56 & & & & & & Gonzales (1994) \\
\hline \multicolumn{11}{|l|}{ Heptapterus } \\
\hline H. sp. & S. Quinta, SP & UP & & 52 & $22 m+26 s m+4 s t$ & 100 & & & & Vissotto (1995) \\
\hline \multicolumn{11}{|l|}{ Imparfinis } \\
\hline I. piperatus & R. São João, SP & UP & & 56 & $56 \mathrm{~m}, \mathrm{sm}$ & $112 *$ & & & & Vicente et al. (1994) \\
\hline I. cf. piperatus & R. Juquiá, SP & UP & & 56 & $24 m+12 s m+20 s t$ & (92) & & & & Fenocchio (1993) \\
\hline I. sp. & b. Canta Galo, SP & UP & & 58 & & & & & & Fenocchio (1993) \\
\hline \multirow[t]{2}{*}{ I. mirini } & b. Jacutinga, S. Quinta, SP & UP & & 58 & $22 \mathrm{~m}+34 \mathrm{sm}+1 \mathrm{~m}+1 \mathrm{~m}$ & 116 & & & ZZ/ZW & Vissotto et al. (1997) \\
\hline & & & & & $22 m+34 s m+1 m+1 s m$ & 116 & & & & \\
\hline
\end{tabular}


number also proved to be conservative with regard to the $\mathrm{FN}$, as the majority of species have an $\mathrm{FN}=96$ (Table I).

The variability in chromosome number was greater among genera of the family Rhamdiidae. Thus, in Pimelodella $2 \mathrm{n}$ varied from 46 to 58 (Almeida-Toledo et al., 1992; Dias and Foresti, 1993; Swarça, 1998), in Imparfinis sp. there were 56-58 chromosomes (Fenocchio, 1993; Vicente et al., 1994; Vissotto et al., 1997) and in Rhamdia the number varied from 58 to 63 because of the presence of B chromosomes (Fenocchio and Bertollo, 1990; Fenocchio, 1993; Vissotto, 1995, Abucarma, 1998). However, $2 \mathrm{n}$ in this genus is most frequently 58 (Table II). The variation of the FN (from 78 to 116) was also greatest among member species of this family. Pimelodella sp., with $2 n=46$, had an intraspecific variation of 78 to 92 in the FN (Toledo and Ferrari, 1976a; Braga, 1989, Vissotto, 1995; Silva et al., 1996; Swarça, 1998). Some of these variations could have been occurring due to different degrees of chromosome condensation leading to different chromosome classifications among authors. Others may be suffering rearrangements, as inversions, leading to a true polymorphism.

In the Pimelodidae family, B chromosomes have been identified in two genera: Bergiaria (Dias and Foresti, 1993) and Iheringichthys (Dias and Foresti, 1990; Vissotto, 1995; Silva et al., 1996). In the family Rhamdiidae, B chromosomes were observed in a species of Pimelodella (AlmeidaToledo et al., 1992) and in almost all of the species of the genus Rhamdia (Fenocchio, 1993; Vissotto, 1995; Maistro, 1996; Abucarma, 1998). This extra chromosome is highly conserved in the latter genus, and is therefore most likely an important characteristic in its karyotypic evolution.

Sexual chromosomes have been observed only in the Rhamdiidae family. In a species of Pimelodella the male individuals are characterized as heterogametic, possessing a sexual chromosomal system of the type XX/XY (Dias and Foresti, 1993). In Imparfinis mirini the females were heterogametic, thus displaying sexual chromosomic system ZZ/ZW (Vissotto et al., 1997).

Although the diploid number in pimelodids is relatively constant, the karyotypic evolution between these two families is more divergent than uniform. The Rhamdiidae family has a greater karyotypic variability than the Pimelodidae family, which corroborates the data of Pinna (1993), who grouped some genera of pimelodids into the new Rhamdiidae family.

\section{ACKNOWLEDGMENTS}

We thank Dr. Oscar Shibatta for reviewing the manuscript. Research supported by CAPES and CNPq.

\section{RESUMO}

No presente trabalho foi realizado um levantamento citogenético de espécies de peixes das famílias Pimelodidae e Rham- diidae, onde foi observado que das 300 espécies pertencentes a estas famílias, apenas 33 foram caracterizadas citogeneticamente. O número diplóide variou de $2 \mathrm{n}=46$ a $2 \mathrm{n}=63$ cromossomos, os cariótipos são freqüentemente assimétricos, com uma predominância de cromossomos do tipo meta/submetacêntricos, resultando portanto, em um número fundamental alto.

\section{REFERENCES}

Abucarma, M. (1998). Caracterização cromossômica de espécies de Rhamdia (Pisces, Pimelodidae) endêmicas da bacia do Iguaçu. Master's thesis, Universidade Estadual de Maringá, Maringá, PR.

Abucarma, M. and Martins-Santos, I.C. (1996). Caracterização cromossômica de duas espécies da família Pimelodidae (Pisces, Siluriformes). Resumos do VI Simpósio de Citogenética Evolutiva Aplicada a Peixes Neotropicais, São Carlos, SP, pp. 73.

Almeida-Toledo, L.F., Foresti, F., Trajano, E. and Toledo Filho, S.A. (1992). Cytogenetic analysis of the Brazilian blind catfish Pimelodella kronei and of its presumed ancestor Pimelodella transitoria. Caryologia 45: 255-262.

Andrade, S.F., Maistro, E.L., Oliveira, C. and Foresti, F. (1998). Caracterização cariotípica da espécie Rhamdia sp. (Pisces, Pimelodidade) proveniente do rio Sapucaí, Represa de Furnas, MG. Resumos do $44^{\circ}$ Congresso Brasileiro de Genética, Águas de Lindóia, SP, pp. 66.

Bigoni, A.P.V., Almeida-Toledo, L.F. and Toledo Filho, S.A. (1992). Estudos citogenéticos em Pseudoplatystoma coruscans (Pimelodidae, Sorubiminae) do rio Mogi-Guaçu, SP. Resumos do IV Simpósio de Citogenética Evolutiva Aplicada em Peixes Neotropicais, Rio de Janeiro, RJ, pp. 32.

Braga, M.A. (1989). Estudos citogenéticos em Pimelodella sp. (Pisces, Siluriformes). Monografia de Bacharelado, Instituto de Biociências, UNESP, Botucatu, SP.

Costa, L.J. and Reggi, R. (1986). Estudos preliminares de duas espécies da família Pimelodidae: Pimelodus maculatus e Parapimelodus valenciennes do rio Guaíba-RS. Resumos do I Simpósio de Citogenética Evolutiva Aplicada em Peixes Neotropicais, São Carlos, SP, pp. 34.

Della-Rosa, V.A., Bertollo, L.A.C., Ferrari, I., Takahashi, C.S., MoreiraFilho, O. and Foresti, F. (1980). Estudos citogenéticos de peixes da Amazônia. II. Ordem Siluriformes. Ciênc. Cult. 32: 735 (Proceedings).

Dias, A.L. and Foresti, F. (1990). Algumas considerações a respeito do cariótipo de Iheringichthys labrosus (Siluriformes, Pimelodidae) do rio Mogi-Guaçu. Resumos do III Simpósio de Citogenética Evolutiva Aplicada em Peixes Neotropicais, Botucatu, SP, pp. 32.

Dias, A.L. and Foresti, F. (1993). Cytogenetic studies on fishes of the family Pimelodidae (Siluroidei). Rev. Bras. Genét. 16: 585-600.

Fenocchio, A.S. (1993). Cromossomos supranumerários no gênero Rhamdia (Pisces). Caracterização cromossômica e considerações sobre a evolução cariotípica nos Siluroidei. Doctoral thesis, Universidade Federal de São Carlos, São Carlos, SP.

Fenocchio, A.S. and Bertollo, L.A.C. (1990). Supernumerary chromosomes in a Rhamdia hilarii population (Pisces, Pimelodidae). Genetica 81: 193-198.

Fenocchio, A.S. and Bertollo, L.A.C. (1992). Karyotype similarities among Pimelodidae (Pisces, Siluriformes) from the Brazilian Amazon region. Cytobios 69: 41-46.

Fenocchio, A.S., Pastorii, M.C., Lopez, P.A., Sanchez, S., Alberdi, A.J., Bordenave, S. and Dib, M.C. (1994). Levantamento citogenético em peixes de água-doce da Argentina: resumo das espécies estudadas. Resumos do V Simpósio de Citogenética Evolutiva Aplicada a Peixes Neotropicais, Botucatu, SP, pp. 8.

Garcia, R.M.G., Sachete, S. and Martins-Santos, I.C. (1990). Aspectos citogenéticos de Iheringichthys labrosus (Pisces, Pimelodidae) do rio Paraná, Região de Porto Rico, PR. Resumos do III Simpósio de Citogenética Evolutiva Aplicada a Peixes Neotropicais, Botucatu, SP, pp. 33.

Gil, H.R. (1993). Características genéticas, moleculares e fisiológicas de Callophysus macropterus (Siluriformes, Pimelodidae) do rio Solimões e do rio Negro (Amazônia Central). Master's thesis, Instituto Nacional de Pesquisas da Amazônia, Universidade Federal do Amazonas, Manaus, AM. 
Gonzales, A. (1994). Estudio preliminar de tres especies pertenecientes al orden Siluriformes (Pisces). Resumos do V Simpósio de Citogenética Evolutiva Aplicada a Peixes Neotropicais, Botucatu, SP, pp. 21.

Hochberg, V.B.M. and Erdtmann, B. (1988). Cytogenetical and morphological considerations on Rhamdia quelen (Pisces, Pimelodidae) - the occurrence of B chromosomes and polymorphic NOR regions. Rev. Bras. Genet. 11: 563-576.

Maistro, E.L. (1996). Caracterização morfológica estrutural de cromossomos supranumerários em peixes. Doctoral thesis, Universidade Estadual Paulista, Botucatu, SP.

Marques, S., Maistro, E.L., Oliveira, C. and Foresti, F. (1998). Estudos cariotípicos na espécie Pimelodus maculatus (Pisces, Pimelodidae) coletada no rio Sapucaí, Represa de Furnas, MG. Resumos do $44^{\circ}$ Congresso Brasileiro de Genética, Águas de Lindóia, SP, pp. 59.

Martins-Santos, I.C., Julio-Jr., H.F. and Burin, I. (1996). Karyotypic studies of four species of the Sorubiminae subfamily (Pisces, Siluriformes). Caryologia 49: 73-80.

Mees, G.F. (1974). Auchenipteridae and Pimelodidae. Zool. Verh. 132: 115-246.

Oliveira, C., Almeida-Toledo, L.F., Foresti, F., Britski, H.A. and Toledo Filho, S.A. (1988). Chromosome formulae of Neotropical freshwater fishes. Rev. Bras. Genét. 11: 577-624.

Pinna, M.C.C. (1993). Higher-level phylogeny of Siluriformes, with a new classification of the order (Teleostei, Ostariophysi). Doctoral thesis, The City University of New York, New York.

Scheel, J.J. (1973). Fish Chromosomes and their Evolution. Interval Report of Danmarks Akvarium, Charlottenlund, Denmark.

Silva, C.T., Dias, A.L. and Giuliano-Caetano, L. (1996). Análise citogenética em Iheringichthys labrosus e Pimelodella sp. (Pisces, Pimelodidae) da Bacia do rio Tibagi. Resumos do VI Simpósio de Citogenética Evolutiva Aplicada a Peixes Neotropicais, São Carlos, SP, pp. 76.

Souza, A.B., Fonseca, C.G., Pinheiro, L.E.L. and Ribeiro,L.P. (1992). Estudos citogenéticos preliminares em Pseudoplathystoma corruscans (Siluriformes, Pimelodidae) da bacia do rio Paraguai. Resumos do IV Simpósio de Citogenética Evolutiva Aplicada a Peixes Neotropicais, Rio de Janeiro, RJ, pp. 28.

Souza, I.L., Moreira- Filho, O. and Castro, R.M.C. (1994). Informações citogenéticas preliminares de uma nova espécie de Pimelodidae cego de cavernas (Itaetê, BA). Resumos do V Simpósio de Citogenética Evolutiva Aplicada a Peixes Neotropicais, Botucatu, SP, pp. 18

Swarça, A.C. (1998). Estudos citogenéticos em peixes das famílias Pimelodidae e Rhamdiidae da bacia do rio Tibagi/PR. Master's thesis, Universidade Estadual de Londrina, Londrina, PR.

Swarça, A.C., Giuliano-Caetano, L. and Dias, A.L. (1999). Cytogenetic characterization through chromosomic banding of Pinirampus pirinampu (Pisces, Pimelodidae) from the Tibagi River basin PR/Brazil. Caryologia 52:31-35

Toledo, V. and Ferrari, I. (1976a). Estudo citogenético de Pimelodella sp. e Rhamdia hilarii (Pimelodinae, Pimelodidae, Pisces): Cromossomo marcador. Científica 4: 120-123.

Toledo, V. and Ferrari, I. (1976b). Estudos citogenéticos de três espécies do gênero Pimelodus (Pisces, Pimelodidae). Científica 4: 101-106.

Valcarcel, A., Brunner, P. and Maggese, M.C. (1993). B-Chromosome polymorphism in South American catfish, Rhamdia sapo. Aquaculture 110: $111-118$.

Vari, R.P. (1992). Systematics of the Neotropical Characiform genus Cyphocharax Fowler (Pisces, Ostariophysi). Smithson. Contrib. Zool. p. 529.

Vasconcelos, C. (1994). Citogenética e polimorfismo cromossômico em espécies da família Pimelodidae (Pisces, Siluriformes). Master's thesis, Universidade de Maringá, Maringá, PR.

Vicente, M.R.R., Margarido, V.P. and Galetti Jr., P.M. (1994). Estudos cromossômicos em Imparfinis piperatus (Pisces, Siluriformes) do Riacho São João (Bacia do Leste). Resumos do V Simpósio de Citogenética Evolutiva Aplicada a Peixes Neotropicais, Botucatu, SP, pp. 17.

Vissotto, P.C. (1995). Estudos citogenéticos na família Pimelodidae (Pisces, Siluriformes). Master's thesis, Instituto de Biociências, Universidade Estadual Paulista, Botucatu, SP.

Vissotto, P.C., Foresti, F. and Oliveira, C. (1997). A ZZ/ZW sex chromosome system in Imparfinis mirini (Pisces, Siluriformes). Cytologia 62: 61-66.

(Received March 9, 1999) 
